\title{
Fall experience and dual-task during gait performance for community-dwelling persons with stroke
}

\author{
Min-Kyu Kim ${ }^{a}$, Eunjeong Kim ${ }^{b}$, Sujin Hwang ${ }^{c}$, Dongwook Son ${ }^{c, d}$ \\ a'Department of Physical Therapy, Graduate School, Sahmyook University, Seoul, Republic of Korea \\ ${ }^{b}$ Department of Physical Therapy, College of Health Science, Dongshin University, Naju, Republic of Korea \\ 'Department of Physical Therapy, Division of Health Science, Baekseok University, Cheonan, Republic of Korea \\ ${ }^{d}$ Department of Physical Therapy, Myongji Choonhey Hospital, Seoul, Republic of Korea
}

Objective: The purpose of this study was to investigate the effects of fall experience and task complexity on gait performance in community-dwelling persons with chronic hemiparetic stroke.

Design: Cross-sectional study.

Methods: Thirty-three persons who had a history of stroke participated in this study. The participants included 18 persons (aged mean 54.0, mean score of 24.6 points on the Montreal Cognitive Assessment, MoCA) with fall experience (faller group) and 15 persons (aged mean 53.7, mean score of 24.7 points on the MoCA) without fall experience (non-faller group) in the previous six months. This study measured balance and gait performance at two different conditions (with/without $70 \%$ of water filled in a 200 cc cup). The participants were clinically assessed using the 10-meter walk test (10MWT), 6-minute walk test (6MWT), Berg Balance scale (BBS), Dynamic Gait Index (DGI), and Timed Up-and-Go (TUG) test.

Results: After analyzation, persons in the faller group performed significantly better on the 10MWT, 6MWT, BBS, DGI, and the TUG test in the no-cup-carrying condition than those in the cup-carrying condition $(p<0.05)$. The persons in the non-faller group also performed significantly better in all outcome measures with the no-cup-carrying condition than those in the cup-carrying condition $(p<0.05)$. However, there was no interaction between fall experience and task complexity in the two groups.

Conclusions: Our results showed that balance and gait performance depended on fall experience and task complexity but fall experience did not interact with task complexity. Clinicians should consider fall prevention and task complexity during therapeutic approaches in persons with hemiparetic stroke.

Key Words: Accidental falls, Gait, Stroke

\section{Introduction}

Survivors following stroke have been reported to have several physical and cognitive problems, including muscle weakness, spasticity, decreased muscle force, abnormal reflexes, visuoperceptual problems, apraxia and so on [1]. Poor posture, which is one of main symptoms, is caused by hemiparesis after stroke and affects falls or injured falls. Falls increase the incidence of fracture on the paretic lower extremity, especially the hip joint, in individuals with hemiparetic stroke. Therefore, falls threaten the performance of daily activities, the ability to integrate into the community, and social roles in stroke survivors. Falls can also lead to reduced functional activities and sedentary lifestyles [1].

It is essential to carry out multi-task performance for the independent life of persons with hemiparetic stroke because all human movements consist of multiple tasks, such as table tasks in a sitting position, hand manipulation, talking with a friend while walking, and preparing food while moving

Received: 25 August, 2018 Revised: 16 September, 2018 Accepted: 17 September, 2018

Corresponding author: Dongwook Son (ORCID https://orcid.org/0000-0002-8562-4132)

Department of Physical Therapy, Division of Health Science, Baekseok University, 76 Munam-ro, Dongnam-gu, Cheonan 31065 , Republic of Korea Tel: 82-41-550-2309 Fax: 82-41-550-2829 E-mail: W.pt8712@gmail.com

(c) This is an Open-Access article distributed under the terms of the Creative Commons Attribution Non-Commercial License (http://creativecommons.org/licenses/ by-nc/4.0) which permits unrestricted non-commercial use, distribution, and reproduction in any medium, provided the original work is properly cited.

Copyright $@ 2018$ Korean Academy of Physical Therapy Rehabilitation Science 
around in the kitchen $[2,3]$. Previous studies have reported that stroke survivors showed more difficulty during the performance of a dual task than during a single task [4-11]. Stroke survivors have been shown to have decreased gait velocity, increase in double support period during gait performance, and poor postural control in cognitive load with motor behavior with dual-task conditions [3-5]. The task complexity would increase the risk of falls in individuals with hemiparetic stroke [3]. However, it is difficult to identify the problems that may arise in performing dual tasks because most clinical tools focus on the ability to perform a single task. The purpose of this study was to investigate the effects of fall experience and task complexity on gait performance in community-dwelling persons with chronic hemiparetic stroke.

\section{Methods}

\section{Participants}

This study enrolled thirty-three persons who had a history of stroke on a voluntary basis from a community rehabilitation center using a brochure that provided information on the study. Inclusion criteria of this study were as

Table 1. Common characteristics of the participants $\quad(\mathrm{N}=33)$

\begin{tabular}{lcc}
\hline \multicolumn{1}{c}{ Characteristic } & $\begin{array}{c}\text { Non-faller group } \\
(\mathrm{n}=15)\end{array}$ & $\begin{array}{c}\text { Faller group } \\
(\mathrm{n}=18)\end{array}$ \\
\hline Sex (male/female) & $9 / 6$ & $12 / 6$ \\
Age (y) & $53.7(15.14)$ & $54.0(9.00)$ \\
Paretic side (right/left) & $9 / 6$ & $12 / 6$ \\
Post-stroke duration (mo) & $23.6(15.87)$ & $25.3(18.68)$ \\
Montreal Cognitive & $24.7(3.31)$ & $24.6(1.38)$ \\
$\quad$ Assessment (points) & & \\
Etiology & $9 / 6$ & $12 / 6$ \\
$\quad$ infarction/hemorrhage) & & \\
\hline
\end{tabular}

Values are presented as number only or mean (SD). followings: a minimum of 6 months following stroke; no neurological disorders except for stroke; ability to ambulate household distances (15 m) with or without an assistive device; no serious visuoperceptual impairment; ability to understand and follow simple verbal instructions; no serious neglect of the paretic limbs. Participants were excluded if they reported serious somatosensory or musculoskeletal disorders. They were classified into either the faller or the non-faller groups according to their self-report of one or more falls in the 6 months before the study [3]. The participants included 18 persons (aged mean 54.0, mean score of 24.6 points on the Montreal Cognitive Assessement, MoCA) with falls experience (faller group) and 15 persons (aged mean 53.7, mean score of 24.7 points on the MoCA) without falls experience (non-faller group) (Table 1).

\section{Procedure}

After providing their informed consent, this study measured balance and gait performance of the subjects during two different conditions of with or without holding a cup filled with water in a $200 \mathrm{cc}$ cup filled up to $70 \%$. The participants were asked to perform the items of five clinical measures at two different conditions. Two physical therapists assessed the five clinical measurement tools of the participants in a calm and well-organized therapy room. The two different conditions were conducted randomly by the participants.

\section{Outcome measures}

The participants were clinically assessed using five clinical measures: the 10-meter walk test (10MWT), 6-minute walk test (6MWT), Berg Balance scale (BBS), Dynamic Gait Index (DGI), and the Timed Up-and-Go test (TUG). The 10MWT and DGI were used to examine the gait performance of the participants. The 10MWT measured walking ability used each participant's highest speed $(\mathrm{m} / \mathrm{s})$ and the DGI was used quantify dynamic balance abilities and

Table 2. Faller group with or without $70 \%$ water in 200 cc cup

$(\mathrm{N}=18)$

\begin{tabular}{lccc}
\hline Characteristic & Without $70 \%$ water condition & With 70\% water condition & $\mathrm{t}(p)$ \\
\hline TUG test (s) & $36.2(18.25)$ & $42.5(25.27)$ & $-3.320(0.004)$ \\
10MWT (s) & $37.4(15.19)$ & $45.2(22.29)$ & $-4.273(0.001)$ \\
6MWT (cm) & $11,184.6(5,290.60)$ & $9,491.6(4,548.12)$ & $6.851(<0.001)$ \\
BBS (score) & $40.6(9.02)$ & $36.4(10.75)$ & $8.154(<0.001)$ \\
DGI (score) & $11.3(4.00)$ & $7.6(4.29)$ & $11.848(<0.001)$ \\
\hline
\end{tabular}

Values are presented as mean (SD).

10MWT: 10-meter walk test, 6MWT: 6-minute walk test, BBS: Berg Balance scale, DGI: Dynamic Gait Index, TUG: Timed Up-and-Go. 
Table 3. Non-faller group with or without $70 \%$ water in $200 \mathrm{cc}$ cup

\begin{tabular}{lccc}
\hline Characteristic & Without $70 \%$ water condition & With $70 \%$ water condition & $\mathrm{t}(p)$ \\
\hline TUG test (s) & $18.4(8.04)$ & $20.6(9.46)$ & $-4.056(0.001)$ \\
10MWT (s) & $18.1(7.53)$ & $19.9(8.81)$ & $-4.537(<0.001)$ \\
6MWT (cm) & $21,414.4(6,675.92)$ & $19,934.4(6,478.48)$ & $5.592(<0.001)$ \\
BBS (score) & $49.5(4.67)$ & $45.1(6.20)$ & $6.326(<0.001)$ \\
DGI (score) & $16.7(3.24)$ & $11.9(3.67)$ & $9.091(<0.001)$ \\
\hline
\end{tabular}

Values are presented as mean (SD).

10MWT: 10-meter walk test, 6MWT: 6-minute walk test, BBS: Berg Balance scale, DGI: Dynamic Gait Index, TUG: Timed Up-and-Go.

gait dysfunction $[1,12]$. The 6MWT was used to examine the maximum distance that a participant can walk in $6 \mathrm{mi}-$ nutes, and greater distance can be interpreted as better performance $[1,13]$. The BBS was used to evaluate the static and dynamic postural stability and the TUG was used to examine anticipatory postural control and walking ability $[14,15]$.

\section{Results}

The tables of this study show the effects of fall experience and task complexity in two different conditions, with or without a cup. After analyses, the persons in the faller group performed significantly better on the 10MWT, 6MWT, BBS, DGI, and TUG during the no-cup-carrying condition than those in the cup-carrying condition. The mean scores were 37.4 second, $11,184.6 \mathrm{~cm}, 40.6,11.3$, and 36.2 second in the no-cup-carrying condition and were 45.2 second, $9,491.6 \mathrm{~cm}, 36.4,7.6$, and 42.5 second in the cup-carrying condition, respectively (Table 2 ). The persons in the non-faller group also performed significantly better in all outcome measures in the no-cup-carrying condition than those in the cup-carrying condition. The mean scores were 18.1 second, $21,414.4 \mathrm{~cm}, 49.5,16.7$, and 18.4 second in the no-cup-carrying condition and were 20.6 second, 19,934.4 $\mathrm{cm}, 45.1,11.9$, and 20.6 second in the cup-carrying condition, respectively (Table 3 ). However, there was no interaction between fall experience and task complexity in the two groups.

\section{Discussion}

This study examined the effects of fall experience and task complexity on postural control and gait performance in community-dwelling persons with chronic hemiparetic stroke. According to the analyses of the results of this study, there is the relationship between task complexity and physical performance. Stroke survivors showed more difficulty with maintaining postural stability during the BBS assessment with the cup-carrying condition than in the no-cup-carrying condition. The participants also showed an increased walking velocity and decreased gait distance in the cup-carrying condition more than the no-cup-carrying condition. This study evaluated the differences between fall experience and task complexity but fall experience did not interact with task complexity in persons with chronic hemiparetic stroke.

Several researchers have reported the relationship between balance and dual-task performance in older adults or persons with neurological diseases [16-19]. McCulloch et $a l$. [19] studied to examine the relationship between postural stability, attention, and dual-task performance in those with acquired brain injury. They reported that balance was more strongly related to fall history and multiple tasks reduced safety levels and made motor performances slower. They also suggested that use of dual-task approaches to identify risk of falling after brain injury [19]. Another study investigated the effects of visual restriction and unstable base dual-task training (VUDT) on postural stability and attention of persons with chronic stroke [18]. The study reported that VUDT was effective in improving postural balance and attention in persons with stroke. In summary of these previous studies, dual-task performance is a fall risk and therefore, dual-task training can be beneficial for improving balance performance and attention after brain injury. The results of this study also showed reduced postural stability and anticipatory postural control abilities during dual-task performance more than single task performance according to the balance and gait evaluations after stroke.

Several contextual components of walking behavior on the over-ground environment, such as the task and environ- 
mental demands, should be considered, along with individual physical abilities. Dual-task interference is another risk factor of participation restriction in daily activities in community-dwelling persons after stroke [10]. A systematic review with meta-analysis reported that dual-task gait training may improve dual-task gait velocity after stroke, but the clinical significance is unclear [10]. A randomized controlled trials reported that cognitive and motor dual task gait training can enhance dual task gait performance after stroke [20]. This study showed that dual-task created reduced physical abilities than single task while gait performance was evaluated using clinical gait measurement tools. Therefore, this study, based on the analyzing corrected data, suggests that dual-tasks have the beneficial effects as a clinical tool to investigate the physical factor of postural stability and gait performance.

Although this study did use reliable and valid clinical tools, this study did not use any clinical technology of kinematic or kinetic parameters for measuring the effects of fall experience and task complexity. Future studies should use current clinical technology with kinematic or kinetic parameters. The number of participating persons was not large enough to generalize the results for hemiparetic stroke. Our results showed that balance and gait performance depend on the fall experience and task complexity but fall experience did not interact with complexity of task. Clinicians should consider fall prevention and task complexity for therapeutic approach in persons with hemiparetic stroke.

\section{Conflict of Interest}

The authors declared no potential conflicts of interest with respect to the authorship and/or publication of this article.

\section{References}

1. O'Sullivan SB, Schmitz TJ, Fulk GD. Physical rehabilitation. 6th ed. Philadelphia: F.A. Davis Co.; 2014.

2. Yang L, Lam FMH, Liao LR, Huang MZ, He CQ, Pang MYC. Psychometric properties of dual-task balance and walking assessments for individuals with neurological conditions: a systematic review. Gait Posture 2017;52:110-23.

3. Alemdaroğlu E, Uçan H, Topçuoğlu AM, Sivas F. In-hospital predictors of falls in community-dwelling individuals after stroke in the first 6 months after a baseline evaluation: a prospective cohort study. Arch Phys Med Rehabil 2012;93:2244-50.

4. Bowen A, Wenman R, Mickelborough J, Foster J, Hill E, Tallis R. Dual-task effects of talking while walking on velocity and bal- ance following a stroke. Age Ageing 2001;30:319-23.

5. Hyndman D, Pickering RM, Ashburn A. Reduced sway during dual task balance performance among people with stroke at 6 and 12 months after discharge from hospital. Neurorehabil Neural Repair 2009;23:847-54.

6. Kizony R, Levin MF, Hughey L, Perez C, Fung J. Cognitive load and dual-task performance during locomotion poststroke: a feasibility study using a functional virtual environment. Phys Ther 2010;90:252-60

7. Yang L, Lam FM, Huang M, He C, Pang MY. Dual-task mobility among individuals with chronic stroke: changes in cognitive-motor interference patterns and relationship to difficulty level of mobility and cognitive tasks. Eur J Phys Rehabil Med 2018;54:526-35.

8. Tisserand R, Armand S, Allali G, Schnider A, Baillieul S. Cognitive-motor dual-task interference modulates mediolateral dynamic stability during gait in post-stroke individuals. Hum Mov Sci 2018;58:175-84.

9. Timmermans C, Roerdink M, Janssen TWJ, Meskers CGM, Beek PJ. Dual-task walking in challenging environments in people with stroke: cognitive-motor interference and task prioritization. Stroke Res Treat 2018;2018:7928597.

10. Plummer P, Iyigün G. Effects of physical exercise interventions on dual-task gait speed following stroke: a systematic review and meta-analysis. Arch Phys Med Rehabil 2018. doi: 10.1016/j.apmr. 2018.04.009. [Epub ahead of print]

11. Park MO, Lee SH. Effects of cognitive-motor dual-task training combined with auditory motor synchronization training on cognitive functioning in individuals with chronic stroke: a pilot randomized controlled trial. Medicine (Baltimore) 2018;97: e10910.

12. Herman T, Inbar-Borovsky N, Brozgol M, Giladi N, Hausdorff JM. The dynamic gait index in healthy older adults: the role of stair climbing, fear of falling and gender. Gait Posture 2009; 29:237-41.

13. Hwang S, Woo Y, Kim KH, Ki KI. Effects of falls experience on cognitive functions and physical activities in community-dwelling individuals with chronic stroke. Int J Rehabil Res 2013; 36:134-9.

14. Bonnyaud C, Pradon D, Zory R, Bensmail D, Vuillerme N, Roche N. Gait parameters predicted by Timed Up and Go performance in stroke patients. NeuroRehabilitation 2015;36:7380.

15. Blum L, Korner-Bitensky N. Usefulness of the Berg Balance Scale in stroke rehabilitation: a systematic review. Phys Ther 2008;88:559-66.

16. Abuin-Porras V, Villafañe JH, Jiménez-Antona C, Palacios A, Martínez-Pascual B, Rodríguez-Costa. Relationship between attention and balance: a dual-task condition study in children. $\mathrm{J}$ Exerc Rehabil 2018;14:349-55.

17. van het Reve E, de Bruin ED. Strength-balance supplemented with computerized cognitive training to improve dual task gait and divided attention in older adults: a multicenter randomizedcontrolled trial. BMC Geriatr 2014;14:134.

18. Kim D, Ko J, Woo Y. Effects of dual task training with visual restriction and an unstable base on the balance and attention of stroke patients. J Phys Ther Sci 2013;25:1579-82.

19. McCulloch KL, Buxton E, Hackney J, Lowers S. Balance, atten- 
tion, and dual-task performance during walking after brain injury: associations with falls history. J Head Trauma Rehabil 2010;25:155-63.
20. Liu YC, Yang YR, Tsai YA, Wang RY. Cognitive and motor dual task gait training improve dual task gait performance after strokea randomized controlled pilot trial. Sci Rep 2017;7:4070. 